\title{
A SIMPLE METHOD FOR MASS PRODUCTION OF FASCIOLA HEPATICA MIRACIDIA ${ }^{1}$
}

As part of a current study on the immunogenic effect of irradiated metacercariae in rats and its potential for use in cattle and other species, a simple and inexpensive method was devised for mass production of fluke miracidia for infecting a large number of snails for metacercariae production.

Fasciola hepatica eggs may be obtained at the slaughterhouse from the gall-bladder of cattle infected with fascioliasis. The gall-bladders are slit open and the egg-containing bile collected in a gallon beaker. The beaker is filled with tap water. The fluke-eggs and bile debris settle to the bottom in about 15 minutes. The supernatant then is carefully decanted, saving the bottom half-inch and then repeated until the supernatant fluid appears clear.

The eggs, with some liver debris, are resuspended in glass-distilled water and filtered through a 100-gauge (149 M) sieve. The "clean" eggs are then spread in a $21 \mathrm{~cm}$. finger bowl, covered with aluminum foil, and placed in a refrigerator at $5^{\circ}-6^{\circ} \mathrm{C}$. The eggs are viable for a year in this state, but it is advisable to use them within the first 6 months.

To induce embryonation, transfer some eggs to sterile Erlenmeyer flasks (125 ml.), add about $125 \mathrm{cc}$. of glass-distilled water, shake gently, and let stand for 10 to 15 minutes. The supernatant fluid is then siphoned off until about $50 \mathrm{cc}$. remains in the flasks. An antibiotic-antifungal solution is added so that the final concentration per ml. of the water contains penicillin $10^{2}$ units, streptomycin $10^{2} \mu \mathrm{g}$. and amphotericin B "Fungizone" $1 \mu \mathrm{g}$.

Cap the flasks with aluminum foil; punch pin-holes in the foil-caps. Wrap individual flasks in carbon-copy paper to exclude light, but not airtight, and place upright below a lighted $60 \mathrm{~W}$. electric bulb set about 12 to 14 inches above the surface upon which the flasks are placed. The heat of the bulb so arranged will raise the temperature of the water in the flasks to about $30^{\circ} \mathrm{C}$.

Shake the flasks gently each day to prevent the eggs from clumping. On the eighth or ninth day, the miracidia are well developed inside the eggs and ready to hatch. Remove the flasks at such time, to a drawer or box where they should be kept at room temperature. Shake the flasks carefully every other day. When miracidia are needed, hatching can be stimulated by transfering embryonated eggs to dechlorinated tap water in a watch glass. Mass hatching of the embryonated eggs will follow within 5 to 10 minutes.

1 Manuscript submitted to the Editorial Board June 13, 1972. 
In our experience, embryonated eggs have remained viable more than 105 days under these conditions. The antibiotic-antifungal solution prevented growth of bacteria and fungi in the water, also clumping of eggs. Eggs refrigerated at $5^{\circ} \mathrm{C}$. for 5 months, subsequently treated with the antibiotic-antifungal solution and incubated, released a higher number of miracidia (53\%), than the control eggs $(9 \%)$. The miracidia from the treated eggs had 100-percent ability to penetrate Lymnaea cubensis.

$P$. Gomes

A. Velarde

J. Chiriboga

Puerto Rico Nuclear Center

D. de León

Agricultural Experiment Station

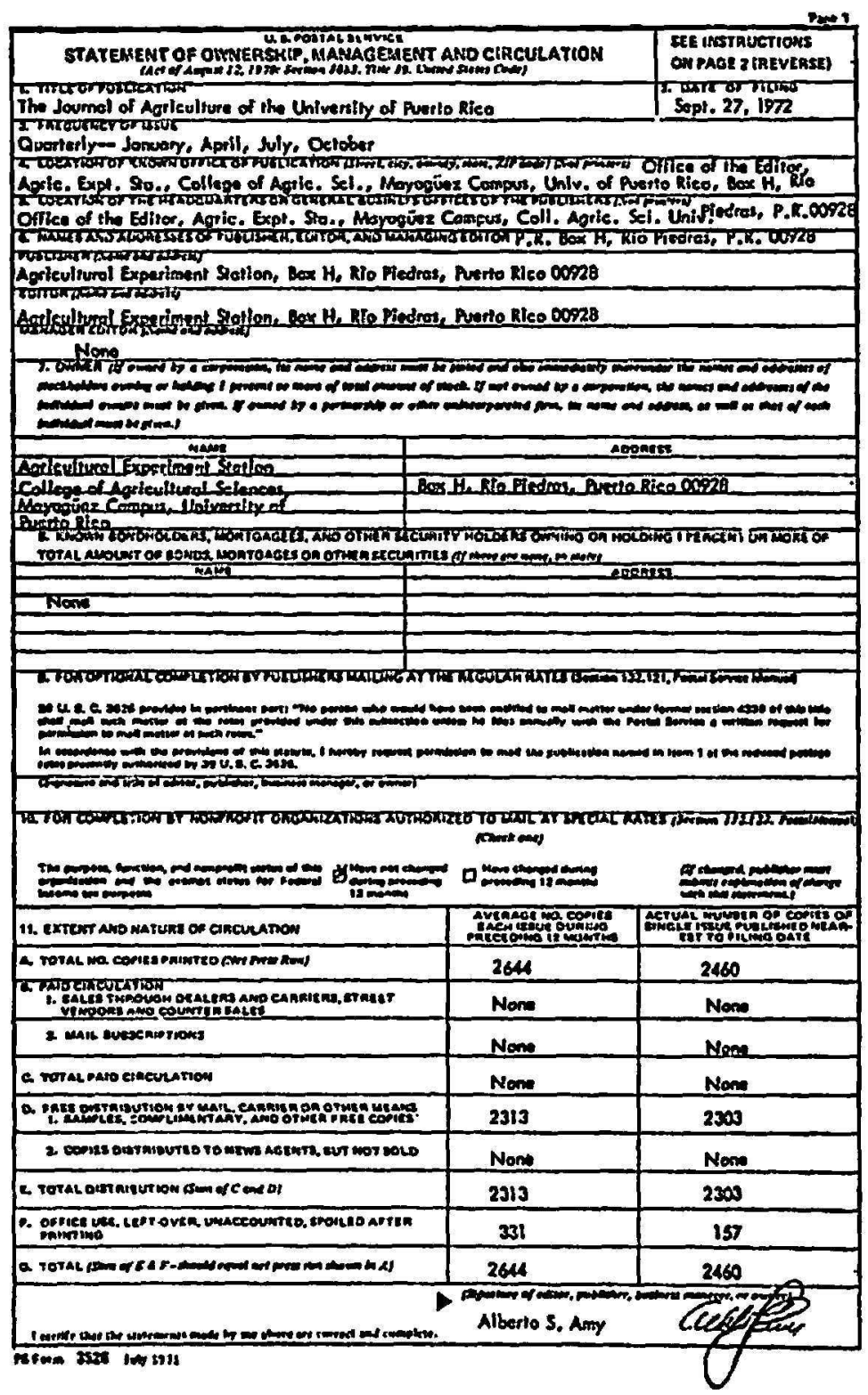

